
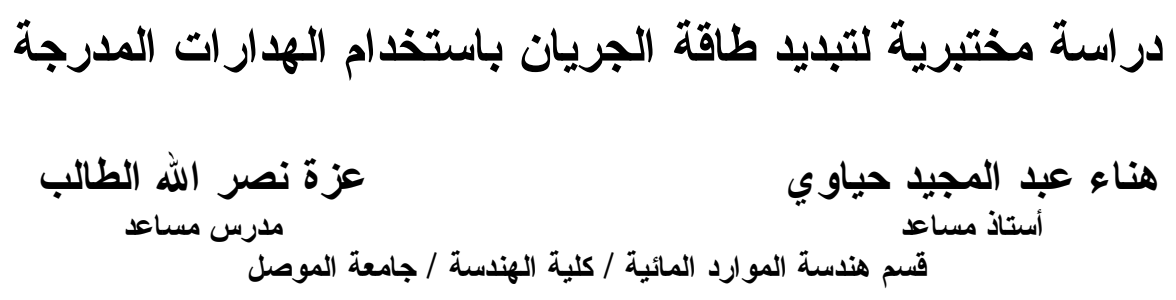

الخلاصة:

أجريت در اسة مختبرية على هدار ات مدرجة وغير مدرجة ، لمعرفة كفاءتها في تبديد طاقة الجريان،حيث تم

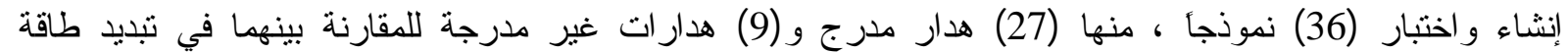

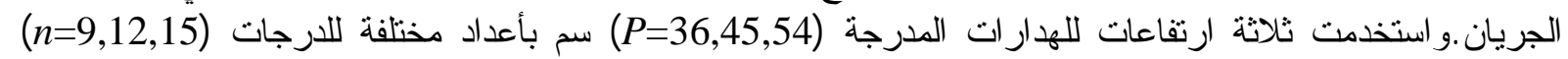

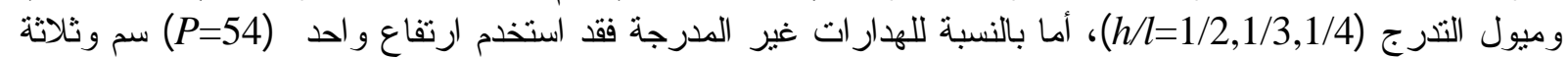

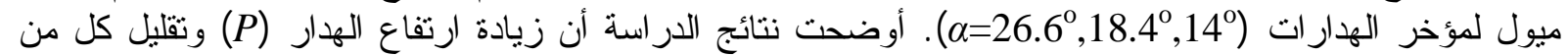

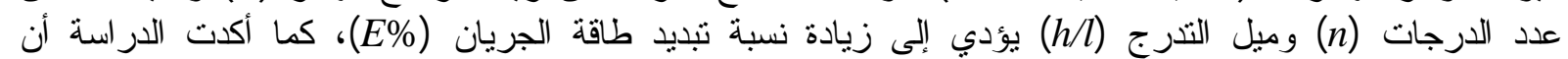

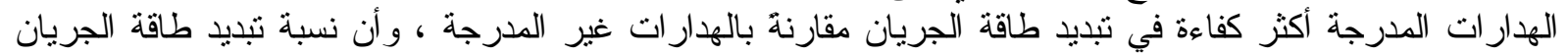

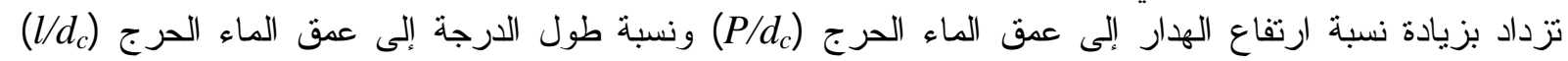

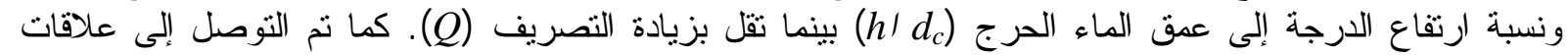

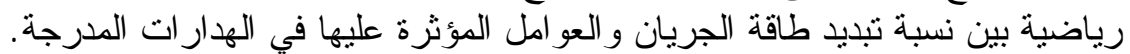
كلمات الدلائة: هدار مدرج، تبذيد طبديد الطاقة.

\title{
Laboratory Study of Flow Energy Dissipation Using Stepped Weirs
}

\author{
Hana Abd AL-Majeed Hayawi \\ Asst. Prof. \\ Azza Nasiralla AL-Talib \\ Asst.Lect.
}

Dept. of Water Resources, College of Eng., Mosul Univ.

\begin{abstract}
A laboratory study was conducted on stepped and unstepped weirs in order to find their efficiency of dissipating flow energy. Thirty six weirs were constructed and tested to compare between stepped and unstepped weirs in flow energy dissipation, twenty seven of them were stepped while the rest were unstepped. Three heights of stepped weirs $(P=36,45,54) \mathrm{cm}$ were used with different numbers of steps $(n=9,12,15)$, and three downstream slopes of stepped face $(h / l=1 / 2,1 / 3,1 / 4)$ were tested, while for unstepped weirs one height of weir $(P=54) \mathrm{cm}$ and three downstream slops $\left(\alpha=26.6^{\circ}, 18.4^{\circ}, 14^{\circ}\right)$ were tested. The results showed that increasing height of weir and decreasing both number of steps and downstream slops stepped face of the weir will cause an increase of the ratio of flow energy dissipation, and the stepped weirs are more efficient in flow energy dissipation compared with unstepped weirs. The percentage of flow energy dissipation $(E \%)$ is increased by increasing the ratio of height of weir to critical water depth $\left(P / d_{c}\right)$, the ratio of length of the step to critical water depth $\left(l / d_{c}\right)$ and the ratio of height of step to critical water depth $\left(h / d_{c}\right)$ while it decreases by increasing the discharge $(Q)$. Imperical relationships between the ratio of flow energy dissipation and factors affecting it were obtained in stepped weirs.
\end{abstract}




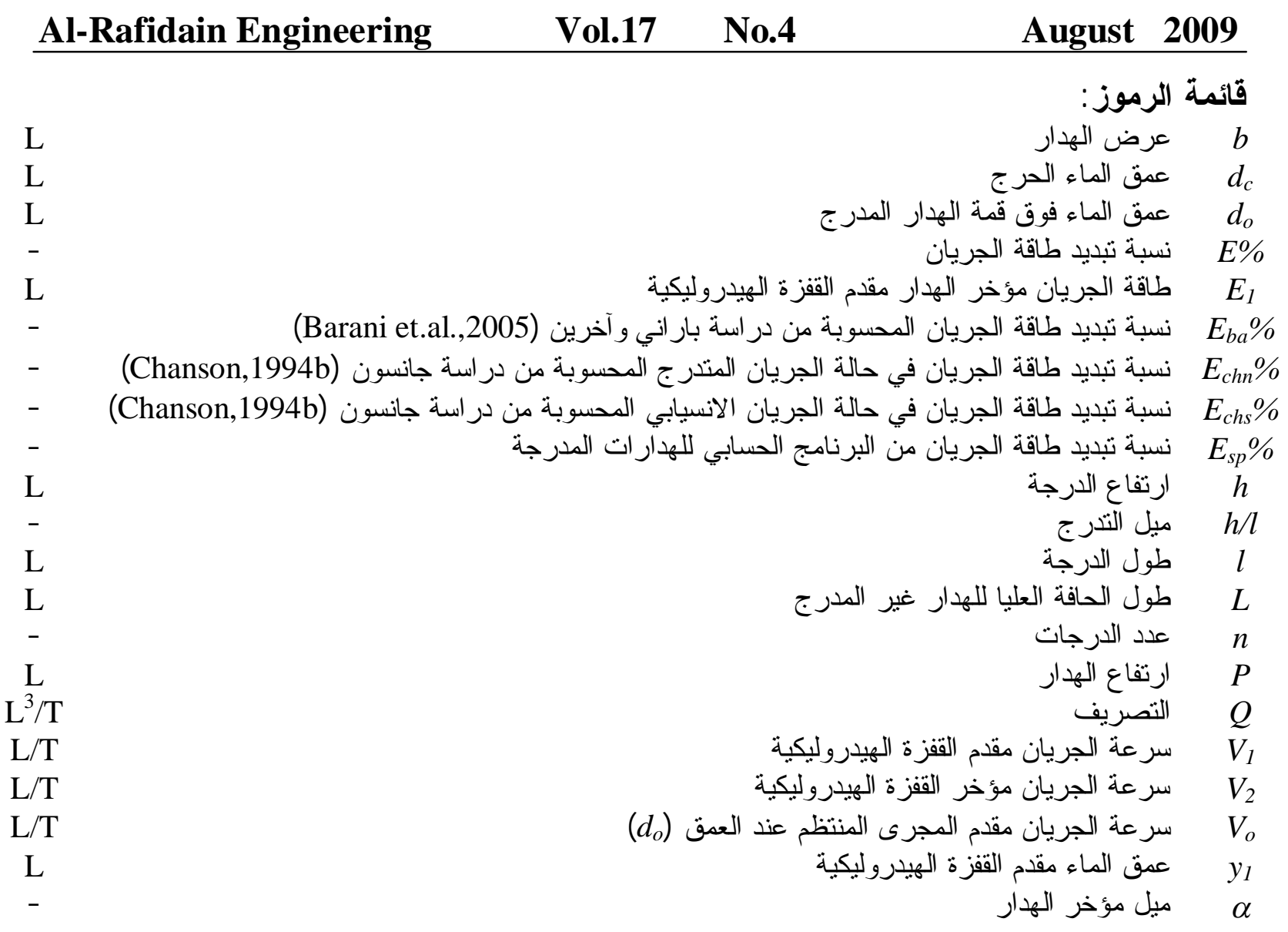

المقدمة:

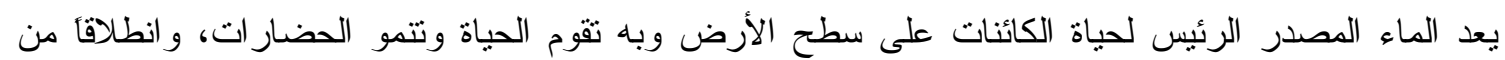

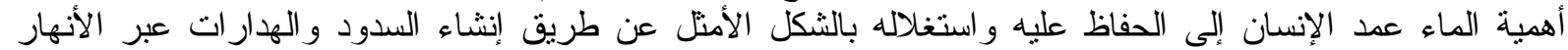

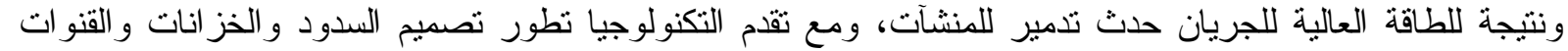

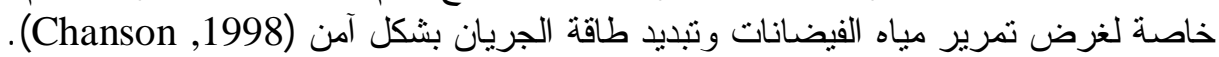

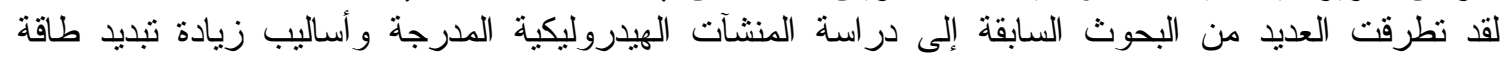

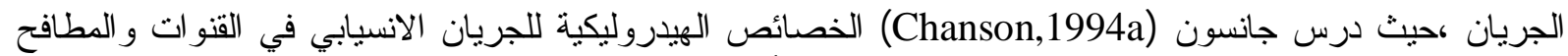
المدرجة ،ولغرض المقارنة بين حالات الجريان المختلفة أجريت دراسة في قناة مدرجة من قبل الباني الباحث جانسون Kumar ( ) (Chanson,1994b)

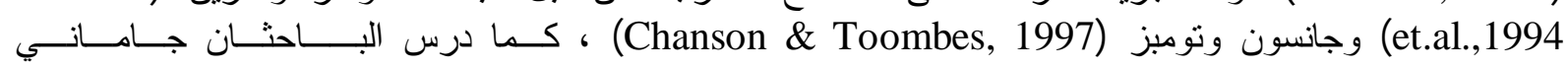

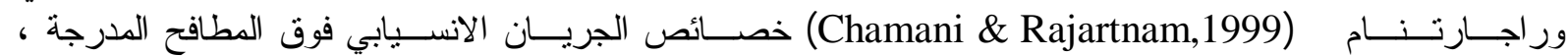

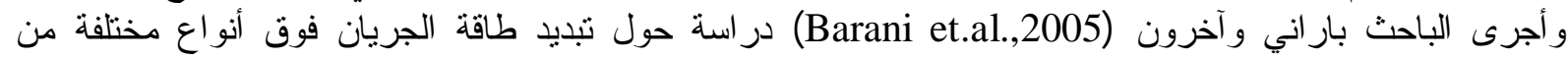
المطافح المدرجة. ومن خلال مر اجعة البحوث السابقة، تبين عدم وجود دراسات مستقيضة حول تغيير الأبعاد الهندسية للهادار المدرج

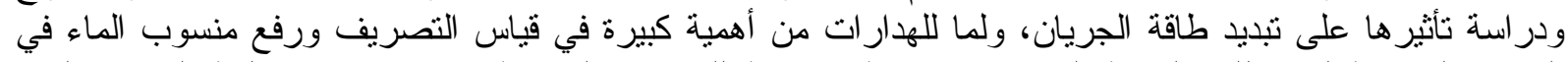

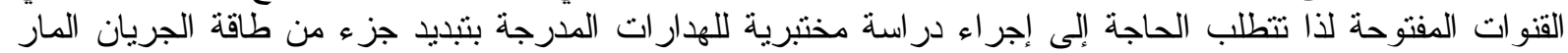
فوقها. لذا نم في هذا البحث اجر اء دراسة مختبرية على هدار ات مدرجة وغير مدرجة ، لمعرفة كفاءتها في تبديد طاقة لكانة

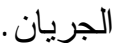

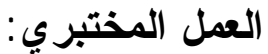

أجريت التجارب في قناة كونكريتية مسنطيلة المقطع بطول (24.64) م وعرض (0.81) م و وعمق (0.76) م يوجد في نهايتها هدار حديدي بأبعاد ( 18 سم ارتفاع ، 50 سم طول، 6 ملم سمك) ،ويوضح الشكل (1) (1) القناة المختبرية المستخدمة في الدر اسة. 


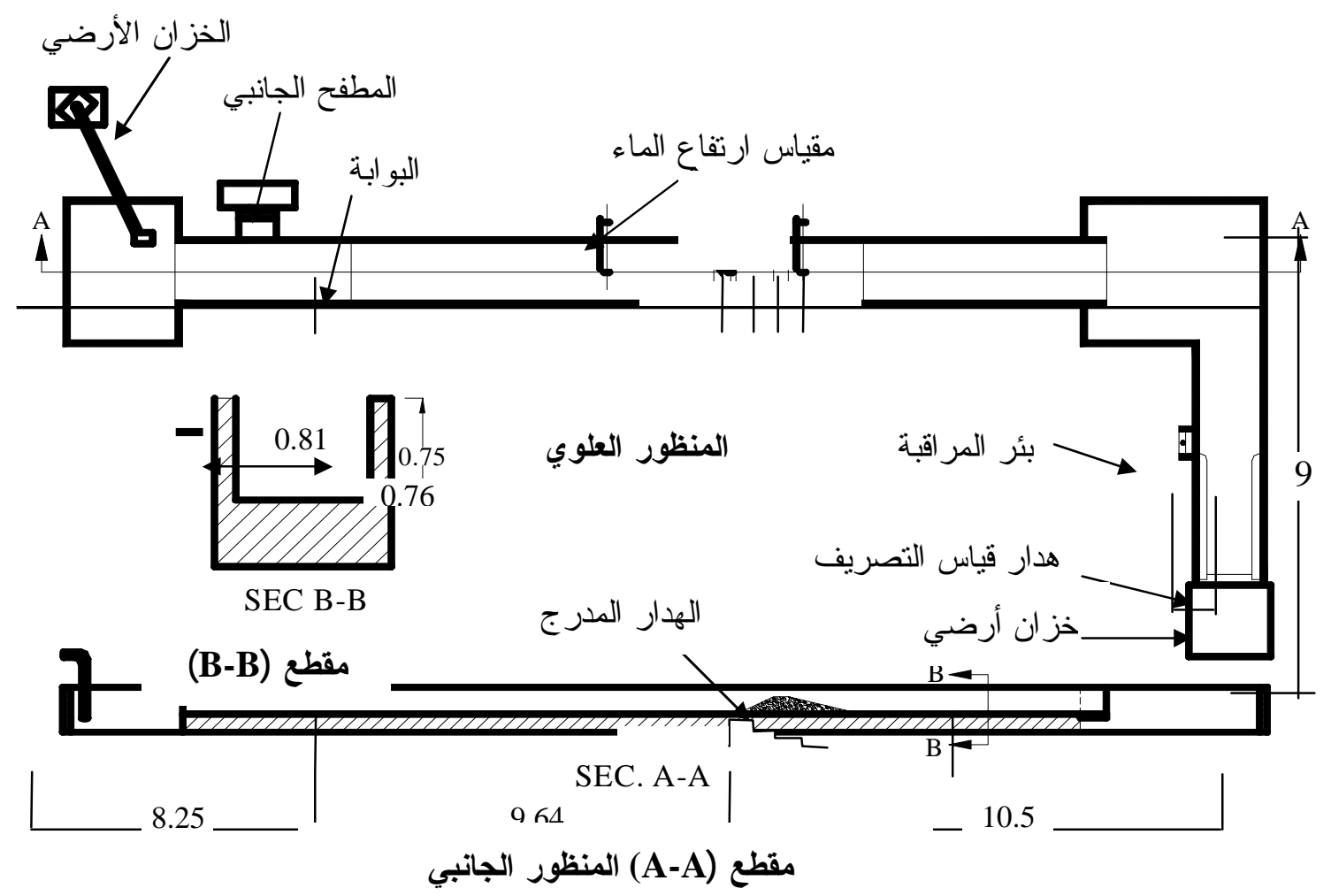

شكل (1) القناة المختبرية جميع الأبعاد بالمتز

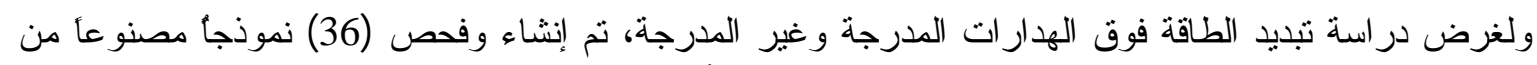

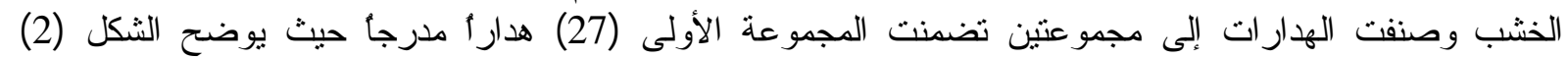

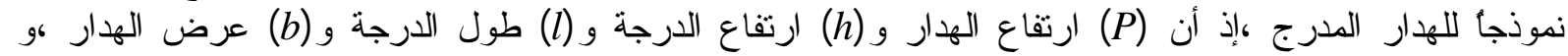

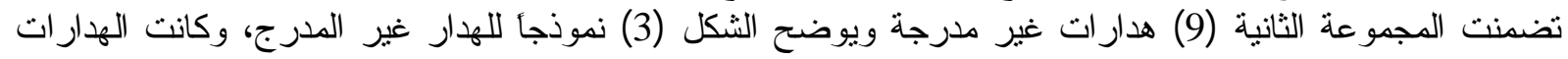

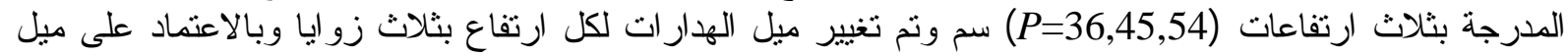

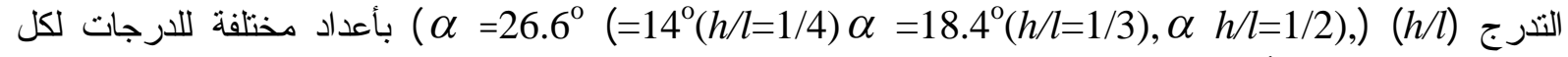

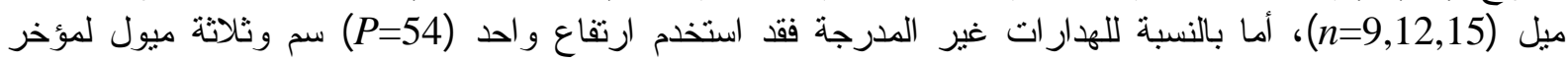
الهدار ات ( )

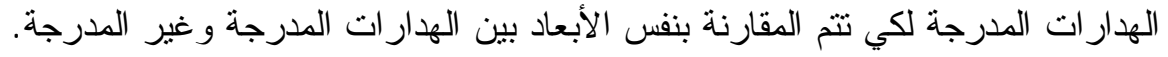

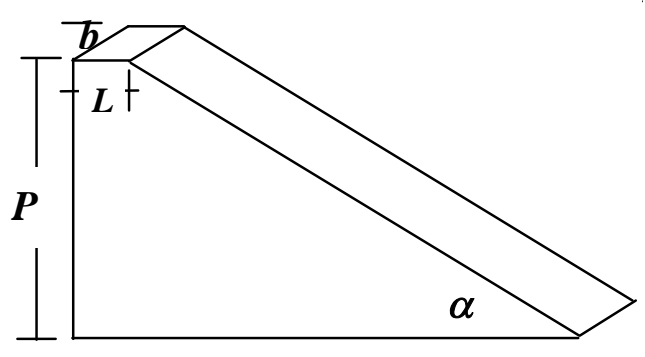

شكل (3) نموذج لهدار غبر مدرج

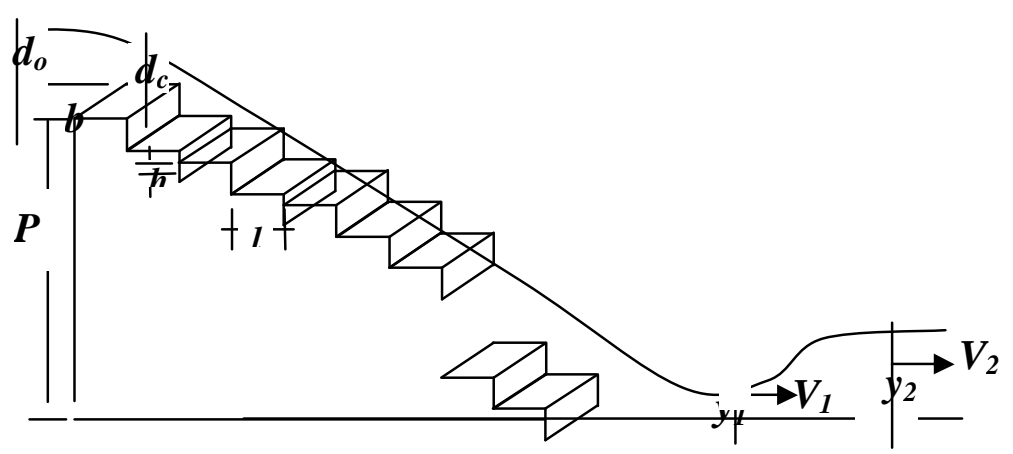

شكل (2) نموذج لهدار مدرج 


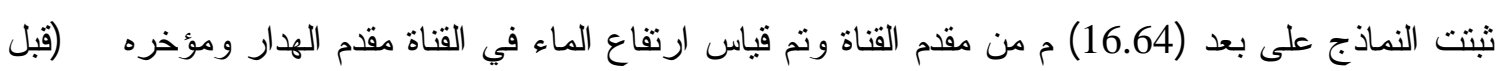

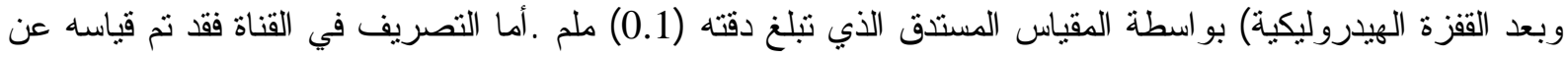

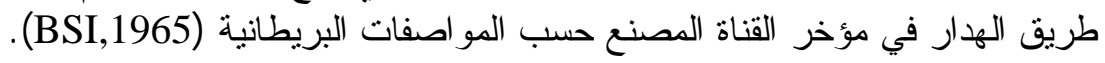

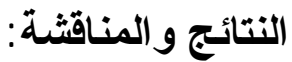

تعرف نسبة تبديد طاقة الجريان بأنها النسبة بين فرق طاقة الجريان مقدم ومؤخر الهدار الددرج إلى طإقاقة الجريان الكلية مقدم الهدار المدرج، وتهدف الدر اسة إلى زيادة نسبة تبديد طاقة الجريان بغية نقليل خطر طاقة الجريان

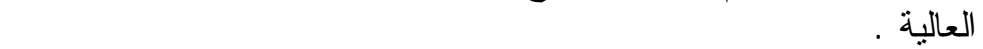
تتأثر نسبة تبديد طاقة الجريان (E\%) للهدارات المدرجة بعدة عوامل منها الخصائص الهندسية للهدار

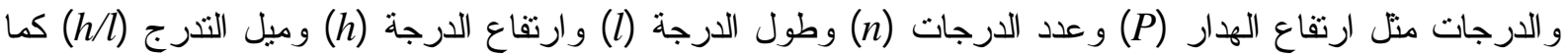

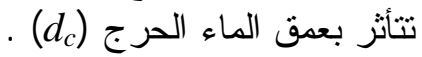
وبإنباع عملية التحليل ألبعدي باستخدام نظرية باي ( Theorem $\pi$ (-) نجد أن نسبة تبديد طاقة الجريان تعد

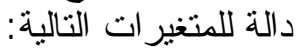

$$
E \%=f\left(\frac{P}{d_{c}}, n, \frac{l}{d_{c}}, \frac{h}{d_{c}}, \frac{h}{l}\right)
$$

أما بالنسبة للهدار ات غير المدرجة فان نسبة تبديد طاقة الجريان تعد دالة للتتغيرات الآتية:

$$
\text { ...(2) } E \%=f\left(\frac{P}{d_{c}}, \frac{L}{d_{c}}, \alpha\right)
$$

Q

L تم حساب نسبة تبديد طاقة الجريان (E\%) كما ليأئ

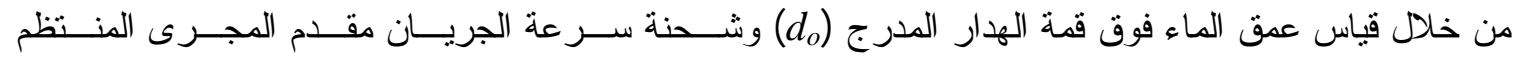

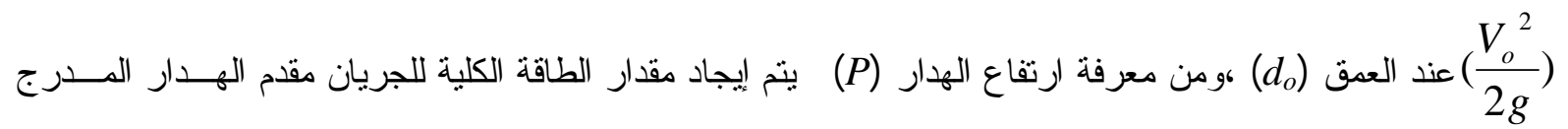
$E_{\max }=P+d_{o}+\frac{V_{o}^{2}}{2 g}$ : وكما يأتي $\left(E_{\max }\right)$

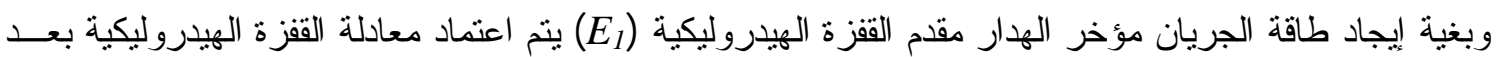

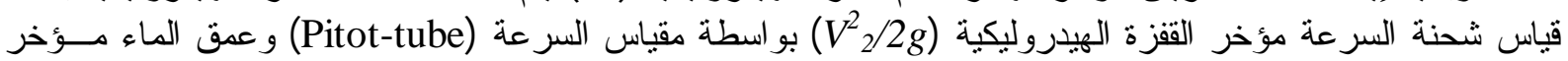

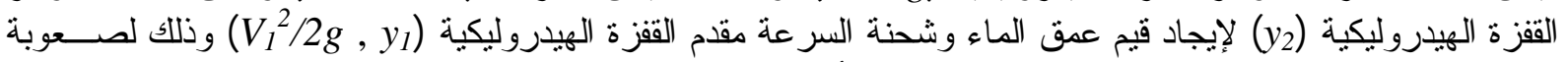
قياسها بسبب اضطر اب الجريان و التهوية العالية وكما يأتي: لإنياء

$$
E_{1}=y_{1}+\frac{V_{1}^{2}}{2 g}
$$

$$
\text { وبعد إيجاد قيم }
$$

$$
E \%=\left(\frac{E_{\max }-E_{1}}{E_{\max }}\right) * 100
$$




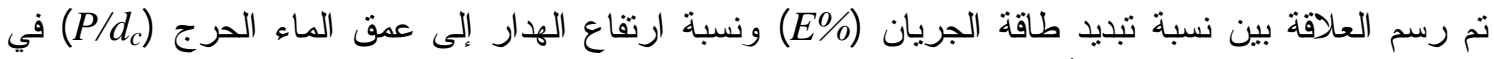

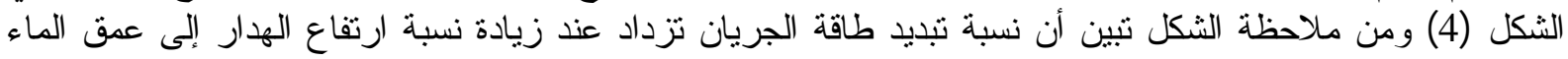

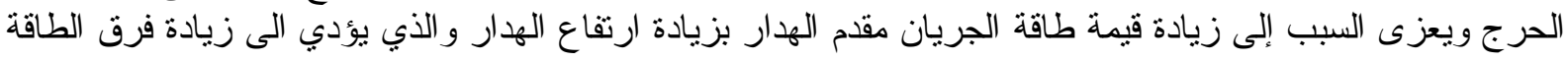
بين مقدم ومؤخر الهدار.

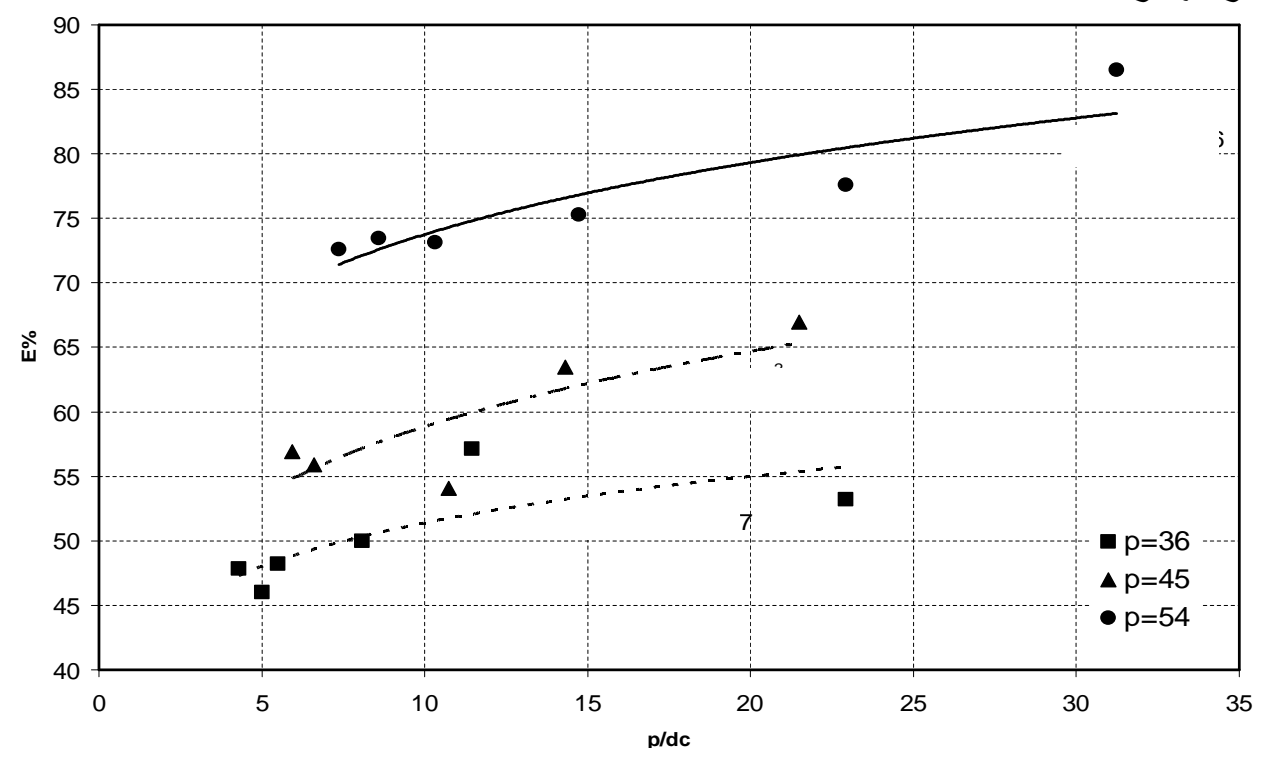

شكل (4) العلاقة بين نسبة تبديد طاقة الجريان ونسبة ارتقاع الهدار إلى عمق الماء الحرج

$$
(n=15, h / l=1 / 4)
$$

العلاقة بين نسبة تبديا طاقة الجريان ونسبة طول الدرجة إلى عمق الماء الحرج:

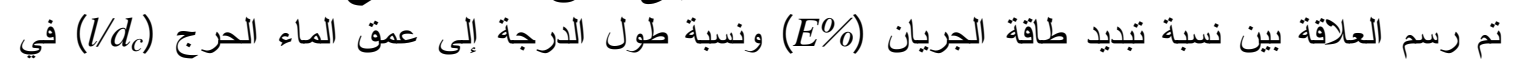

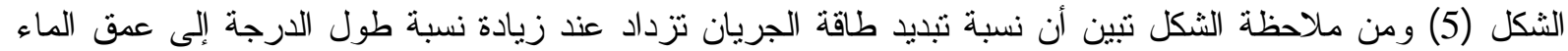

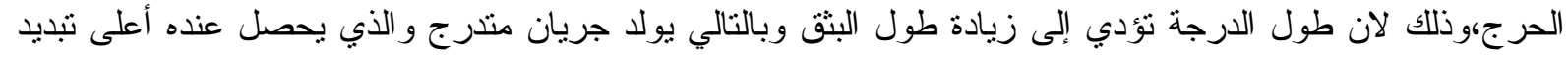

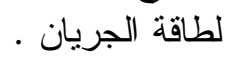

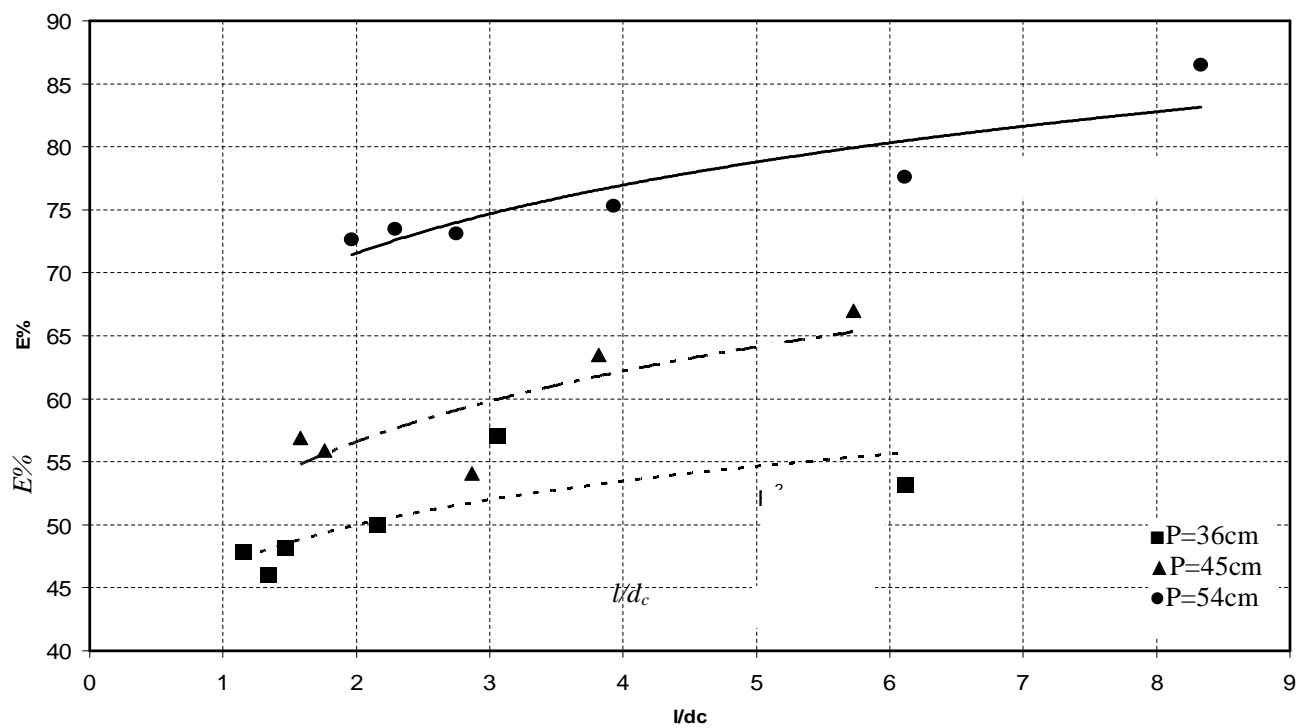

شكل (5) العلاقة بين نسبة تبديد طاقة الجريان ونسبة طول الدرجة إلى عمق الماء الحرج

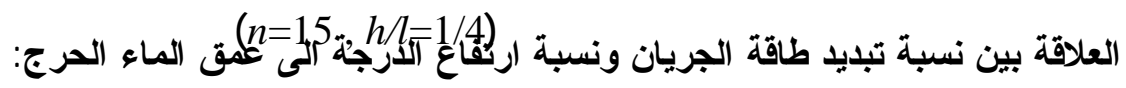




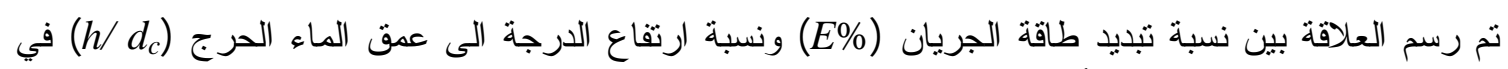

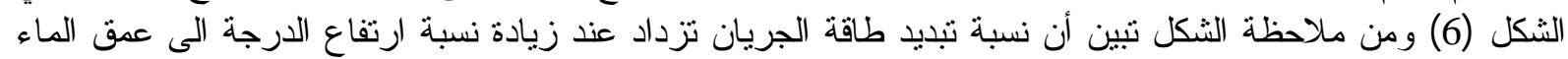

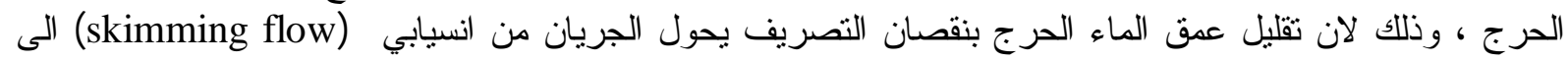
منترج (nappe flow) و الذي تزداد عنده طاقة الجريان المبددة.

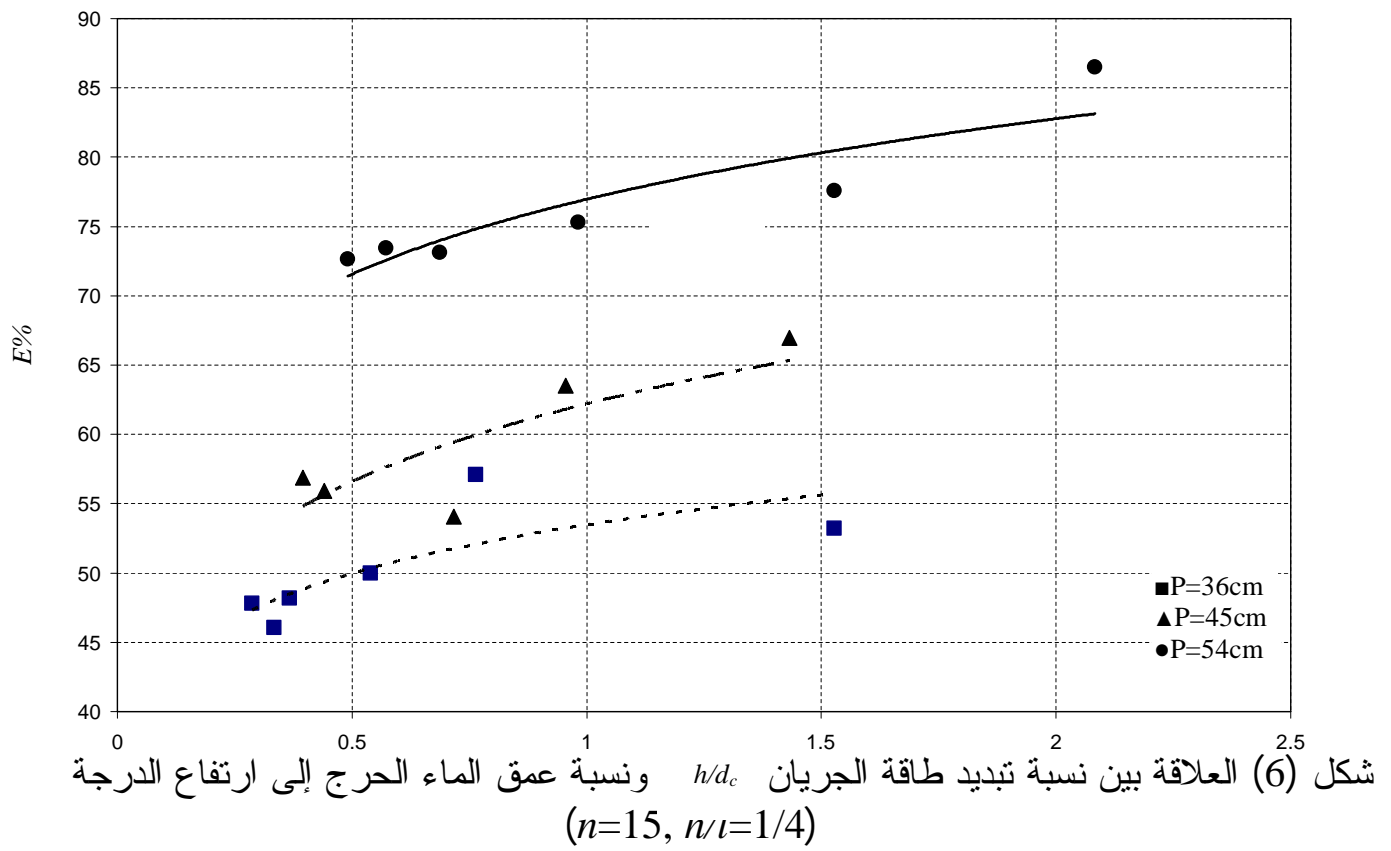

العلاقة بين نسبة تبديد طاقة الجريان و التصريف :

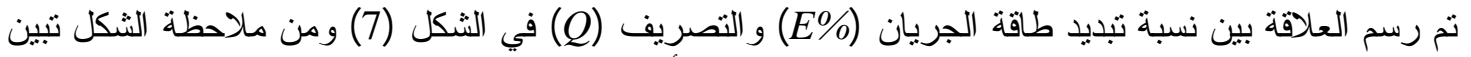

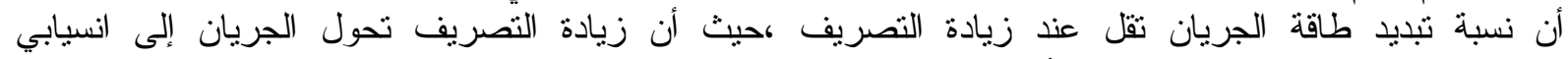

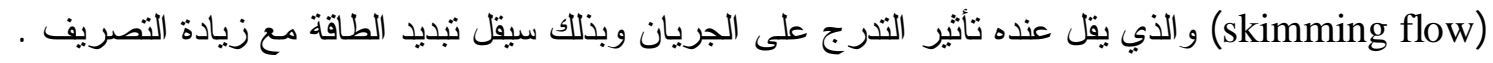

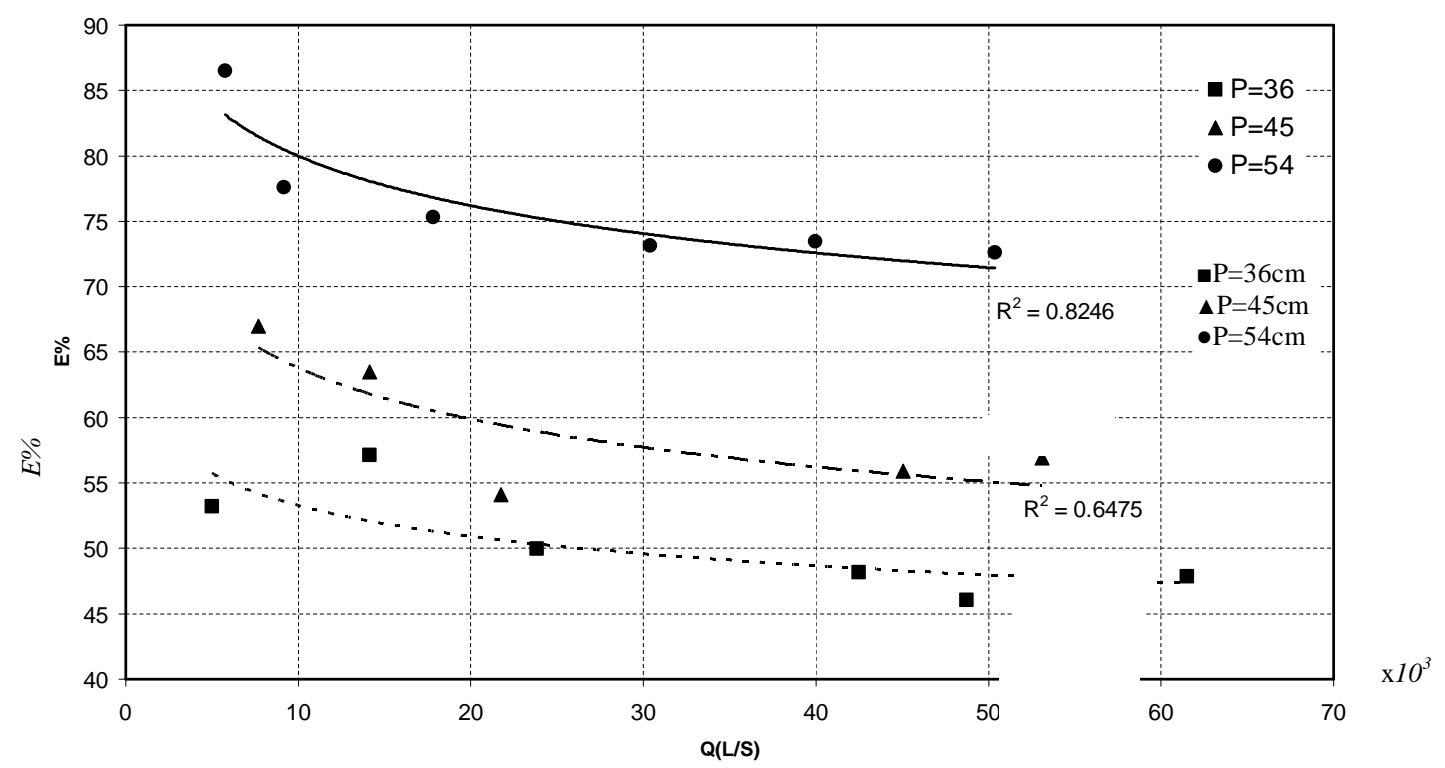

شكل (7) العلاقة بين نسبة تبديد طاقة الجريان و التصريف (n=15,h/l=1/4) 


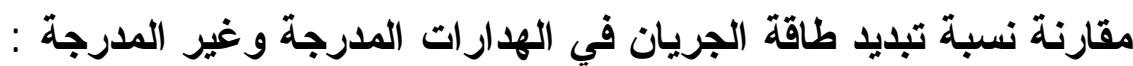

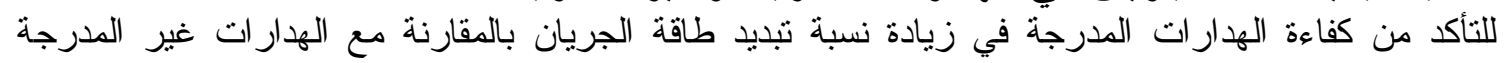

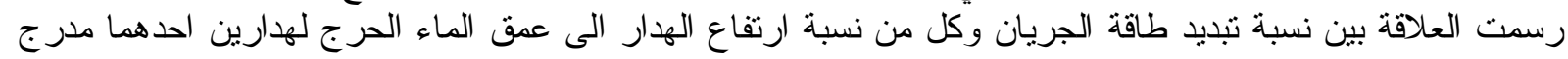

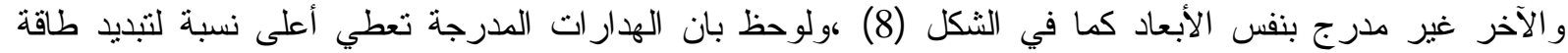

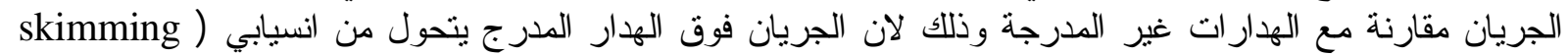

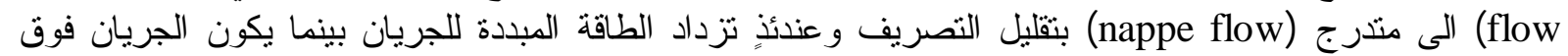
الهدار غير المدرج من النوع الانسيابي فقط .

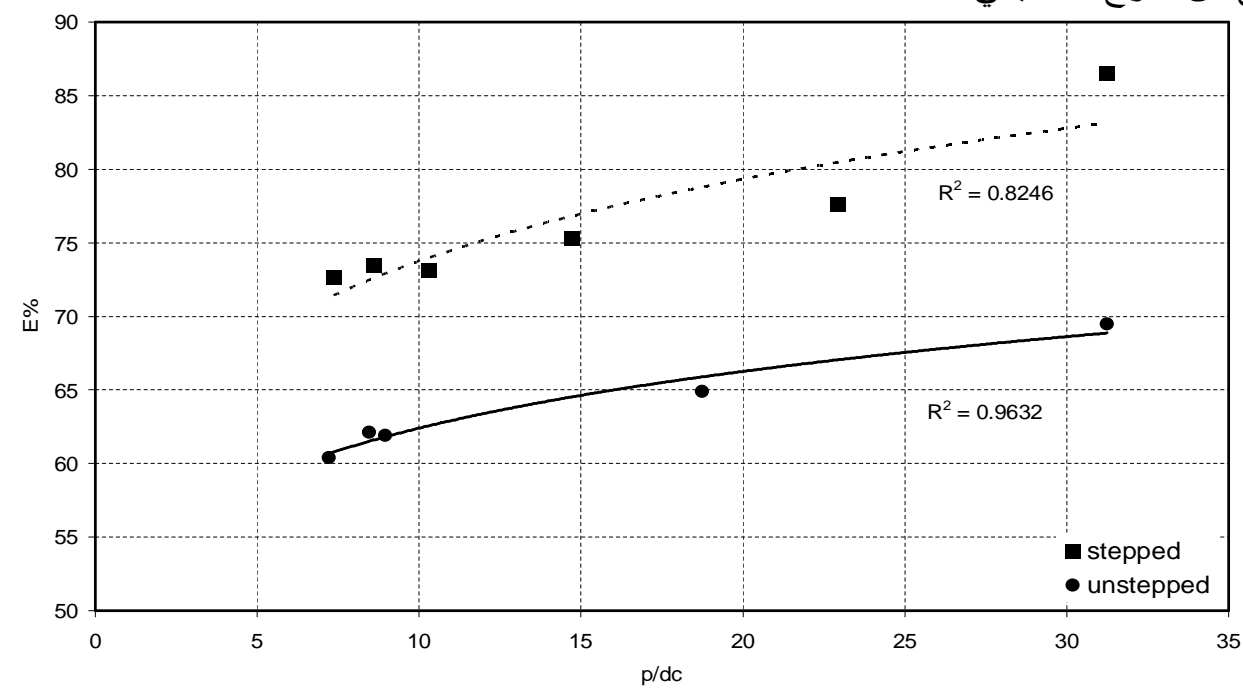

شكل (8) العلاقة بين نسبة تبديد طاقة الجريان ونسبة ارتفاع الهدار إلى عمق الماء الحرج

$$
(P=54 \mathrm{~cm}, n=15, l=14.4 \mathrm{~cm})
$$

تأثير عدد الارجات على العلاقة بين نسبة تبديد طاقة الجريان ونسبة ارتفاع الهدار الى عمق الماء من خلال در اسة تاثير عدد الدرجات على العلاقة بين نسبة تبديد طاقة الجريان ونسبة ارتفاع الهدار الى عمق الماء الحرج الموضحة في الثكل (9)،فان نسبة تبديد طاقة الجريان تزداد كلما قل عدد الدرجات الى الى حد معين ضمن محددات الدر اسة.

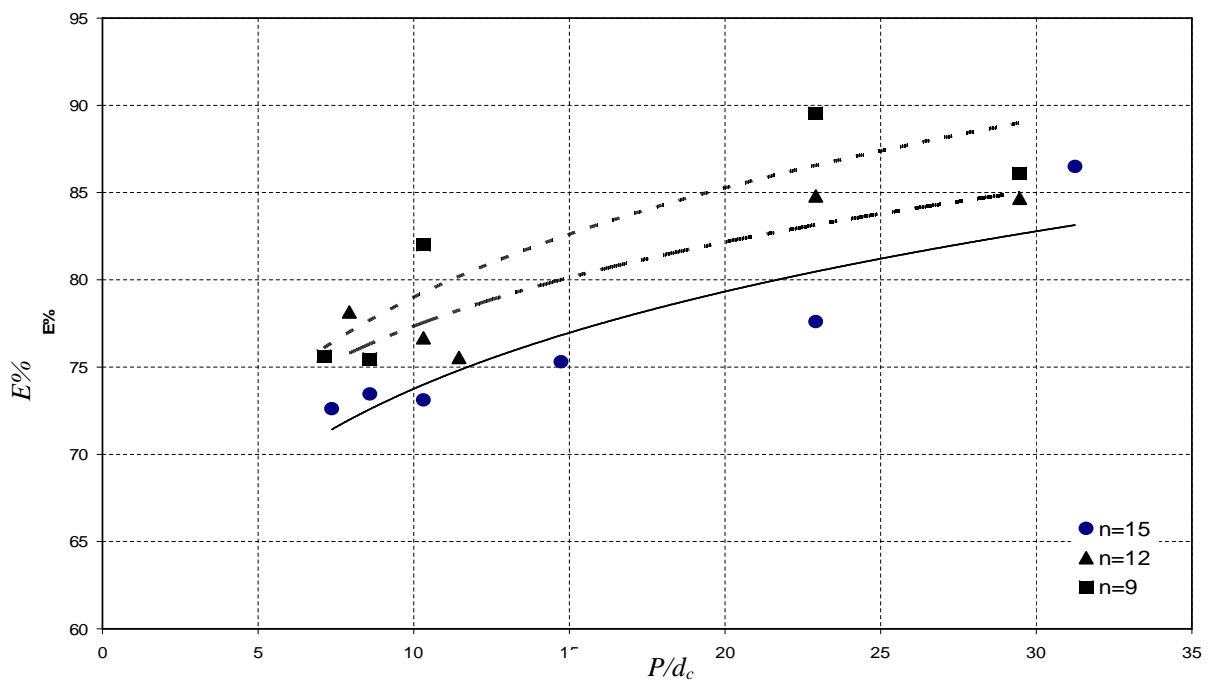

شكل (9) تأثثر عدد الدرجات على العلاقة بين نسبة تبديد طاقة الجريان ونسبة ارتفاع الهدار إلى عمق الماء الحرج $(P=54 \mathrm{~cm}, h / l=1 / 4)$ 
من خلال معرفة العو امل المؤثرة على نسبة تبديد طاقة الجريان في الهذارات المدرجة عن طريق التحليل

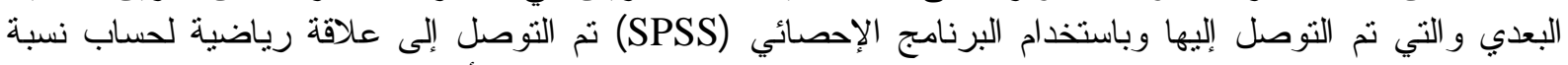

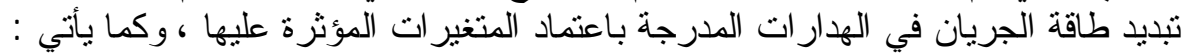

$$
\begin{gathered}
E \%=41.28\left(P / d_{c}\right)^{0.16} \\
E \%=119.52(n)^{-0.268} \\
E \%=50.99\left(l / d_{c}\right)^{0.18} \\
E \%=62.12\left(h / d_{c}\right)^{0.17} \\
E \%=51.89(h / l)^{-0.16}
\end{gathered}
$$

ومن خلال كافة المتغير ات المؤثزة على نسبة تبديد طاقة الجريان فان العلاقة كما يلي:

$E_{s p} \%=206.64\left(P / d_{c}\right)^{5.67}(n)^{-6.3}\left(l / d_{c}\right)^{-34}\left(h / d_{c}\right)^{28.57}(h / l)^{-34.24}$

بلغ معامل الارتباط للمعادلة العامة أعلاه (0.7).

$$
\text { \% E Esp }
$$

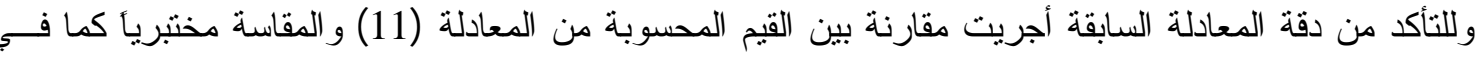

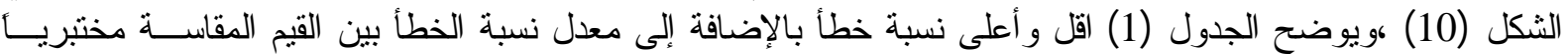

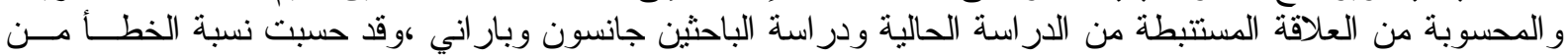

\begin{tabular}{|c|c|c|c|c|}
\hline \multicolumn{3}{|c|}{ الدر اسات السابقة } & الدر اسة الحالية & \multirow{2}{*}{ القيم المحسوبة } \\
\hline$E_{b a} \%$ & $E_{\text {chs }} \%$ & $E_{c h n} \%$ & $E_{s p} \%$ & \\
\hline$\% 0.06$ & $\%$ \%2.7 & \%0.33 & $\% 0.13$ & أقل نسبة خطأٔ\% \\
\hline \%99.8 & \%75.4 & \%82.8 & $\% 43.4$ & أعلى نسبة خطأه\% \\
\hline$\% 48.5$ & \%34.2 & $\% 33$ & $\% 14.5$ & معدل نسبة الخطأة\% \\
\hline
\end{tabular}

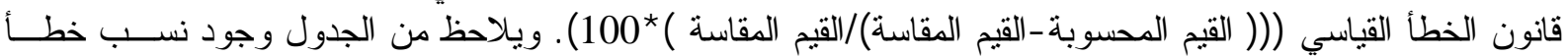
عالية عند مقارنة البحوث السابقة ويعزى ذللك إلى اختلاف محددات الدراسة من حيث الميول المستخدمة و التصــاريف

جدول (1) نسب الخطأ بين قيم نسبة تبديد طاقة الجريان

المقاسة مختبرياً و المحسوبة من المعادلة (7) نباندان

إذ أن:

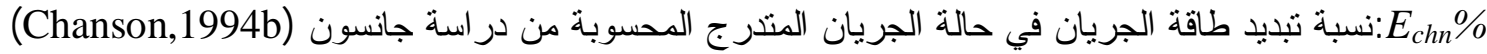

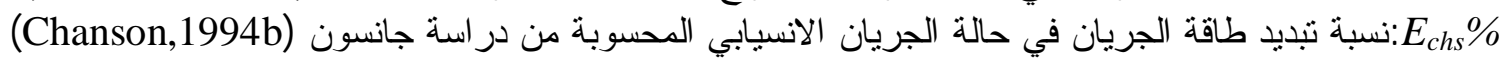

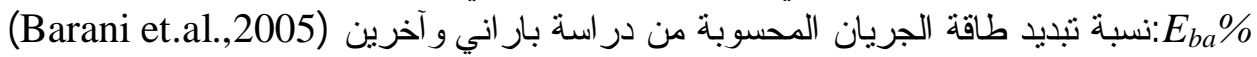




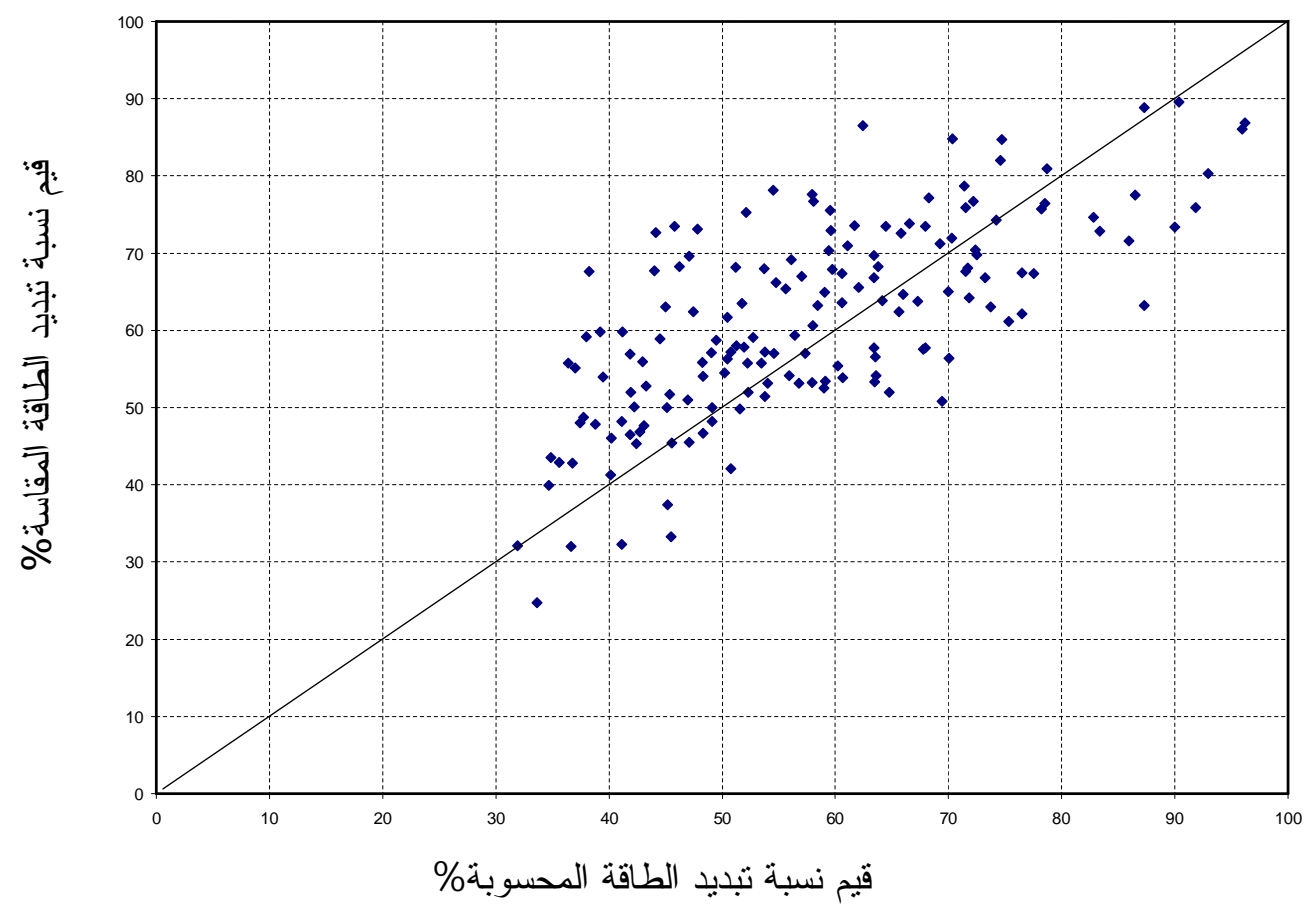

شكل (10) العلاقة بين نسبة تبديد طاقة الجريان المقاسة مختبريأ و المحسوبة من الدراسة الحالية

1 - تزداد نسبة تبديد طاقة الجريان للهدارات المدرجة بزيادة ارتفاع الهدار ونسبة ارتفاع الهدار إلى عمق الهاء

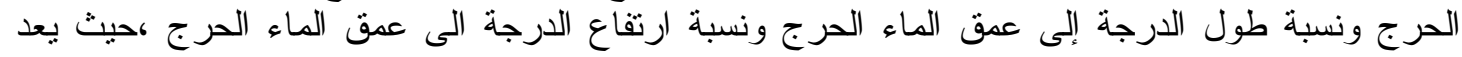

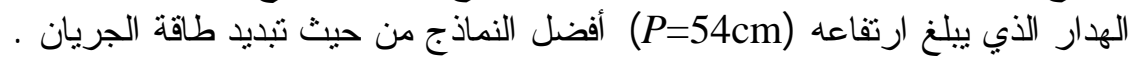

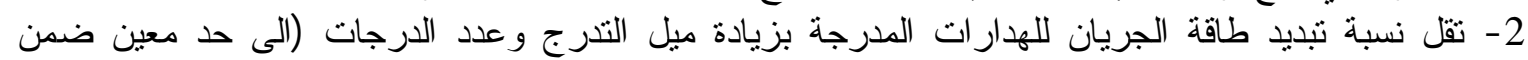

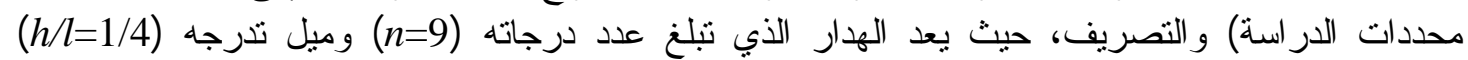

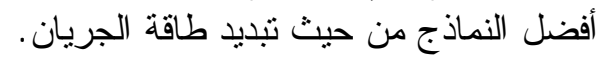
3 - تعد الهدار ات المدرجة أكثر كفاءة من الهدار ات غيدة الهربان المدرجة في تبديد طاقة الجريان.

1. Barani, G.A., Rahnama, M.B., Sohrabipoor, N., 2005, "Investigation of Flow Energy Dissipation over Different Stepped Spillways", American. Journal of Applied Sciences, 2(6), 1101-1105.

2. British Standard Institution (BSI),1965,"Thin-plate Weirs and Venturi Flume in Methods of Measurements of Liquid Flow In Open Channel",Part4A,BSI,3681,London.

3. Chamani, M.R., Rajaratnam, N.,1999,"Characteristics of Skimming Flow over Stepped Spillways" Journal of Hydraulic Engreering, ASCE,125(4),361-368.

4. Chanson, H., Toombes, L.,1997."Energy Dissipation in Stepped Waterway" Proce. XXVII IAHR, the27 th Congress,595-600.

5. Chanson, H.,1994a,"Hydraulics of Skimming Flows over Stepped Channels and Spillways", Journal of Hydraulic Researches, IAHR,32(3),445-460. 


\section{\begin{tabular}{llll} 
Al-Rafidain Engineering & Vol.17 & No.4 & August 2009 \\
\hline
\end{tabular}}

6. Chanson, H.,1994b,"Comparison of Energy Dissipation Between Nappe and Skimming Flow Regimes on Stepped Chutes", Journal of Hydraulic Researhes, IAHR, 32 (2),213218.

7. Chanson, H.,1998,"Review of Studies on Stepped Channel Flows", Workshop on Flow Characteristics around Hydraulic Structures and River Environment, Nihon Univ., Tokyo, Japan.

8. Kumar, D.N., Nayak, P.C., Pillai, C.R.S.,1994,"Model Investigations of Stepped Spillway", http://www. citeseer.csail.mit.edu.

9. Vennard, J.K., Street, R.L.,1982,"Elementary Fluid Mechanics", John Wiley and Sons, Canada, 685. 